\title{
Los materiales biodegradables, una alternativa a la contaminación de los polímeros sintéticos
}

\section{Biodegradable materials, an alternative to the contamination of synthetic poly-}

mers

Pedro Rodríguez Sandoval 1 María Isabel Arévalo2 DOI: $10.29151 /$ reit.n1a3

1. Email: prodirguezs@misena.edu.co, Grupo de investigación Gimes, SENA-Centro de Materiales y Ensayos; Grupo Investigación NIEIT, Unimonserrate, Bogotá, Colombia

². Email:miarevalo0@misena.edu.co, Grupo de investigación Gimes, SENA-Centro de Materiales, Bogotá, Colombia 



\section{Resumen}

En la actualidad se presenta un gran problema por el crecimiento exponencial de los desechos, causado principalmente por la acumulación de plásticos fabricados por la industria y empleados por la sociedad en una amplia línea de artículos para cubrir las necesidades en diferentes áreas, los cuales en su mayoría son de un solo uso, esto los convierte en grandes generadores de contaminación ambiental por su baja degradabilidad y el impacto negativo que estos ocasionan en las fuentes hídricas.

Esta es una de las razones por las que en los últimos años las industrias se han enfocado en la obtención de materiales que resuelvan estos problemas, y es precisamente esto lo que se aborda en el presente proyecto, donde se realizó la caracterización de un material compuesto formado por una mezcla de almidón de papa y polietileno, bajo parámetros establecidos, el cual demostró tener buenas propiedades térmicas haciéndolo apto para la fabricación de artículos industriales. Se evaluó la velocidad de degradación del material durante aproximadamente 6 meses en un ambiente con alta carga de microorganismos; gracias a la hidrolisis, la presencia de humedad en el suelo debilita los enlaces entre las moléculas del material, facilitando que los microorganismos puedan utilizarlo como alimento, generando $\mathrm{CO}_{2}$, agua y biomasa como productos de desecho. Para corroborar la biodegradabilidad del material se tuvieron en cuenta los métodos descritos en las normas ASTM D-6400 y D-5988, en un ambiente que se asemeja a un vertedero de basuras.

Palabras claves: biodegradabilidad; bioplásticos; envases; empaques; proceso inyección. 


\begin{abstract}
Currently there is a great problem due to the exponential growth of waste, caused mainly by the accumulation of plastics manufactured by the industry and used by society in a wide line of articles to cover the needs in different areas, which in their Most are single-use, this makes them great generators of environmental pollution due to their low degradability and the negative impact they cause on water sources, this is one of the reasons why in recent years industries have focused on in obtaining materials that solve these problems, and this is precisely what is addressed in this project, where the characterization of a composite material formed by a mixture of potato starch and polyethylene was carried out, under established parameters, which demonstrated have good thermal properties making it suitable for the manufacture of industrial items. The degradation rate of the material was evaluated for approximately 6 months in an environment with a high load of microorganisms; Thanks to hydrolysis, the presence of moisture in the soil weakens the bonds between the molecules of the material, making it easier for microorganisms to use it as food, generating $\mathrm{CO}_{2}$, water and biomass as waste products. To corroborate the biodegradability of the material, the methods described in ASTM D-6400 and D-5988 standards were taken into account, in an environment that resembles a garbage dump.
\end{abstract}

Keywords: Biodegradability; bioplastics; containers; packaging; injection process. 


\section{Introducción}

Los polímeros sintéticos han sido un valioso aporte al desarrollo industrial y tecnológico del mundo, convirtiéndose en acompañantes habituales de nuestra vida, sin embargo hoy son considerados un problema ambiental por la gran producción de artículos que se convierten en desechos, esto se evidencia en los informes estadísticos de producción en Estados Unidos, Europa occidental y Japón en donde se reportan cifras de 101, 8 millones de toneladas de la cuales 36,4 millones de toneladas están en rellenos sanitarios (2019).

En Colombia el uso de los materiales poliméricos de tipo sintético en los procesos de inyección, soplado, extrusión, termo formado y roto moldeo han incrementado su producción anual, generando que sus ventas se incrementen al compararlos con años anteriores según estudios de Acoplásticos, asociación a la que pertenecen la mayor parte de empresas de este sector. Esta asociación registra que al año se procesan 1.300 .000 toneladas de resinas plásticas por un valor de 4000 millones de dólares al año. Así mismo, se evidencia que el consumo de plásticos por habitante es de 27 kilogramos, de los cuales el 56\% corresponde a productos para empaque, envase y pitillos, el $6 \%$ pertenece a artículos de dotación y línea hogar, y el $22 \%$ elementos de construcción. De dichos elementos, solo se recicla el $17 \%$ ocasionando graves problemas ambientales por la gran cantidad de desechos, razón por la cual las autoridades nacionales y locales se han visto obligadas a tomar disposiciones de orden jurídico, como el decreto ley del año 2008, que regula el uso de las bolsas. La normatividad ambiental y la tendencia actual del mundo por incrementar la conciencia ambiental, está obligando a las industrias a buscar materiales más amigables con el medio ambiente para fabricar sus artículos.
En coherencia con lo anterior, las investigaciones de polímeros biodegradables con base de almidón se iniciaron hacia los años setenta, al respecto, se pueden relacionar los trabajos de investigación del Dr. Contreras Andújar de la Universidad Carlos III de Madrid (2006), los doctores Ortiz \& Villalobos de la Universidad Tecnológica del Suroeste de Guanajuato, México (2013), los doctores Vieyra R.H Y San Martin M.E del centro de investigación de ciencia aplicada y tecnología avanzada del instituto politécnico nacional México (2009),en donde como resultado se obtuvieron materiales biodegradables para procesos primarios de transformación como inyección y extrusión.

En este sentido, el objetivo de esta investigación fue la transformación y evaluación de las propiedades mecánicas y biodegradabilidad de un material compuesto biodegradable, resultante de mezclar almidón de papa con polietileno de baja densidad, para los procesos industriales de inyección y extrusión.

\section{Metodología}

\subsection{Preparación y alistamiento de materia prima}

Para este trabajo de investigación, se utilizó como materia prima el polietileno de baja densidad marca: DOW referencia 9931 para el proceso de extrusión y para el proceso de inyección, polietileno de alta densidad Marca E14412020 KANG Y C DOW referencia 9931, almidón de papa modificado referencia: ALMI 4 procesado por ALMICOR Ltda., aditivos: y agua destilada. El alistamiento de la materia prima se inició con la mezcla del almidón industrial $70 \%$, gliceraldehído al $5 \%$ y agua destilada el $25 \%$. 


\subsection{Proceso de extrusión e inyección}

En esta etapa del proyecto se utilizó de la máquina de extrusión de doble husillo marca: Thermo Scientific referencia: 05 PTW16, con la que se definieron los parámetros de transformación de filamentos para luego pasar a la maquina peletizadora, en donde se obtuvieron los pellets. Se realizaron 4 tratamientos con diferentes porcentajes de almidón (2016).

Posteriormente, se procede al mezclado de los pellets en un dosificador volumétrico para ser alimentado a la maquina inyectora de mono tornillo marca: Wittmann Smart 60 de 60 toneladas de fuerza de cierre y capacidad de inyección 150 gramos, se utilizó un molde de acero de inyección de dos cavidades estandarizado bajo la norma ASTM D 638 de la probeta tipo A corbatín.

\subsection{Caracterización propiedades mecánicas}

Para la caracterización de las propiedades mecánicas se realizó el ensayo de tensión que consiste en someter una probeta a una carga axial, con el fin de que se produzca la rotura de la probeta para determinar la resistencia y las propiedades mecánicas del material, este proceso se desarrolló en la maquina universal marca BESMAK BMT-E con celda de cinco toneladas.

\subsection{Ensayo de biodegradabilidad}

El procedimiento para analizar la degradabilidad del material siguió la guía estándar para evaluar la compostabilidad en el medio ambiente para plásticos degradables, que se muestra en la norma ASTM D-6002. Para ello, se preparó un compost, el cual contenía microorganismos que son favorables para la degradación satisfactoria del material. La preparación del suelo consistía en una mezcla de tierra común y compost, se depositan las probetas protegiéndolas de la luz del sol, posteriormente se monitoreó la evolución de estas probetas cada 15 días, para finalmente cada mes sacarlas y realizar ensayos pertinentes de acuerdo con la norma ASTM D-5988, en la cual se rectifica cualitativamente si el proceso de degradación se está llevando a cabo de manera satisfactoria, con el propósito de determinar el grado degradabilidad del material y el tiempo necesario al igual que condiciones ambientales que favorecen a que la descomposición avance más rápido.

\section{Resultados y Discusiones}

\subsection{Procesos de extrusión e inyección}

En los procesos de transformación con este material compuesto biodegradable se realizaron la programación de la variables según los tratamientos establecidos en el diseño experimental, como los valores de temperaturas, presiones, velocidades, porcentajes de almidón y se tuvieron en cuenta los resultados de las investigación realizados por Ortiz y Villalobos (2013); Rodríguez, Camargo y ViIlagrán (2016); Muñoz, Gómez y Rodríguez (2015) y Rodríguez y Camargo (2016) donde se trabajó con películas, filamentos e inyección de piezas con polietilenos y almidones de carga.

Para el proceso de extrusión se obtuvieron filamentos de $4 \mathrm{~mm}$ de diámetro, los cuales se llevaron a la maquina peletizadora para obtener pellets de $4 \mathrm{~mm}$ de diámetro por $4 \mathrm{~mm}$ de longitud. Por inyección se obtuvieron como producto probetas tipo corbatín con los diferentes tipos de mezclas. Ver figura 1. 


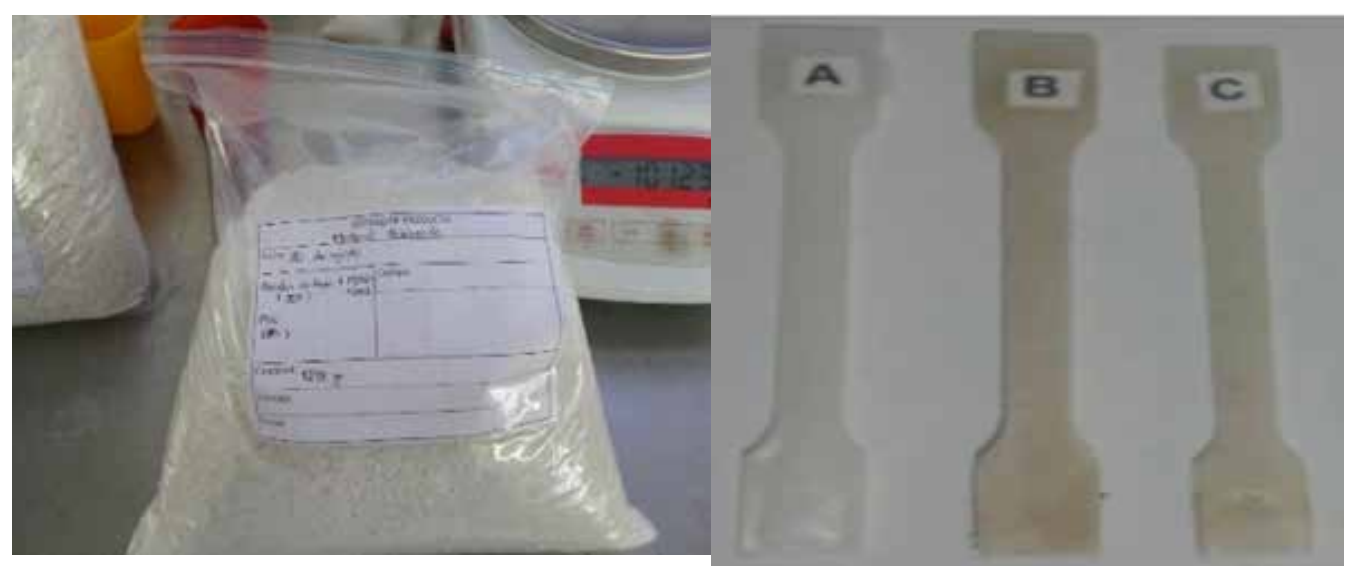

Figura 1: Productos finales pellets y probetas tipo corbatín. Fuente: Autor

\subsection{Caracterización propiedades mecánicas}

\subsection{Ensayos de tensión}

Luego de llevar a cabo el proceso de inyección donde se tomaron cinco muestras diferentes de acuerdo con las concentraciones de almidón de papa industrial, PEBD, los resultados obtenidos en la investigación son:

Se pudo observar que con el PEBD al aumentar el porcentaje de almidón su elongación disminuía, ya que por efecto genera cambios en su estructura química, como se ilustra en la Figura 2.

\section{TRATAMIENTO}

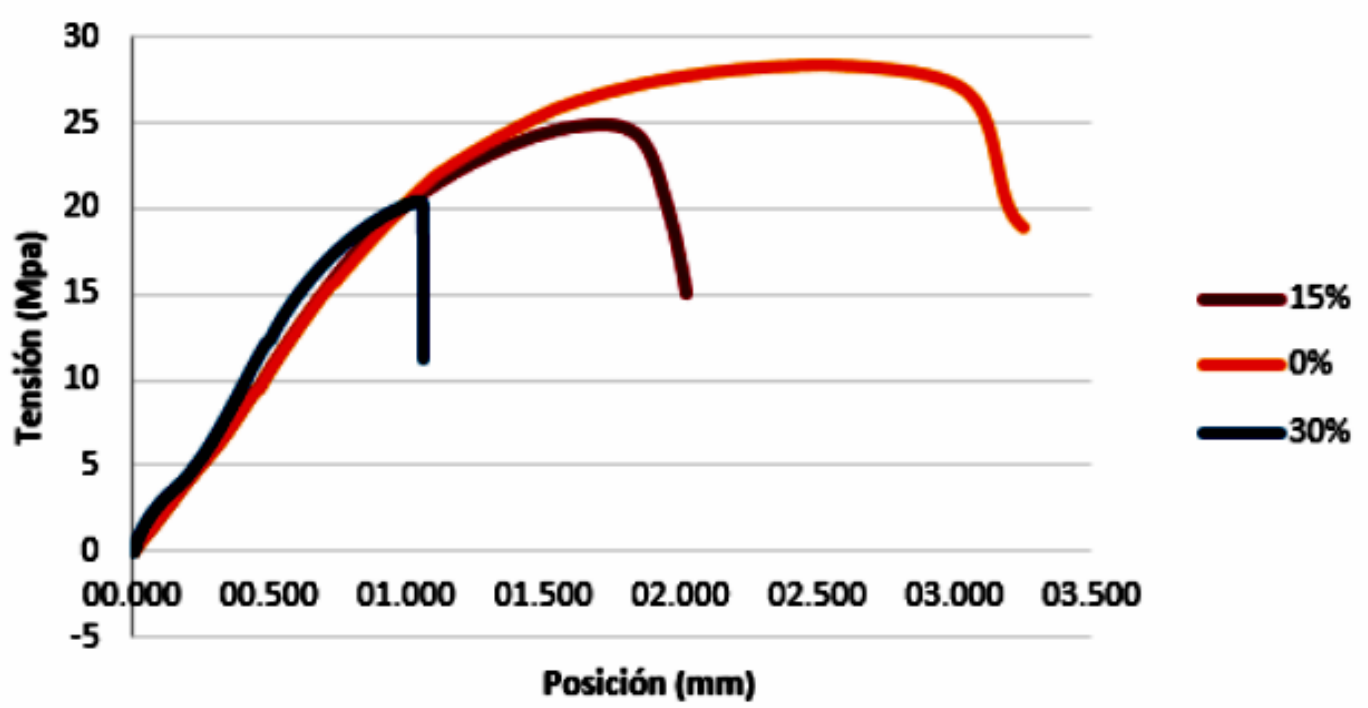

Figura 2: Curva esfuerzo - deformación Fuente: Autor 


\subsection{Ensayos de biodegrabilidad}

Los ensayos de biodegradabilidad se desarrollaron de acuerdo con la norma ASTM D-5988 por medio de la determinación del $\mathrm{CO}_{2}$ el cual es producido durante la degradación causada por los microorganismos al consumir el bioplástico, mostrados en la figura 3 se estableció que el biopolímero es degradable por medios biológicos encontrados en el compost. Además, se evidencia que a medida que se aumenta el contenido de almidón en la matriz de polietileno de baja densidad, la producción de $\mathrm{CO}_{2}$ es mayor; mientras que la producción de $\mathrm{CO}_{2}$ es muy baja con contenidos bajos de almidón, indicando que la biodegradación de un plástico totalmente sintético no es viable.

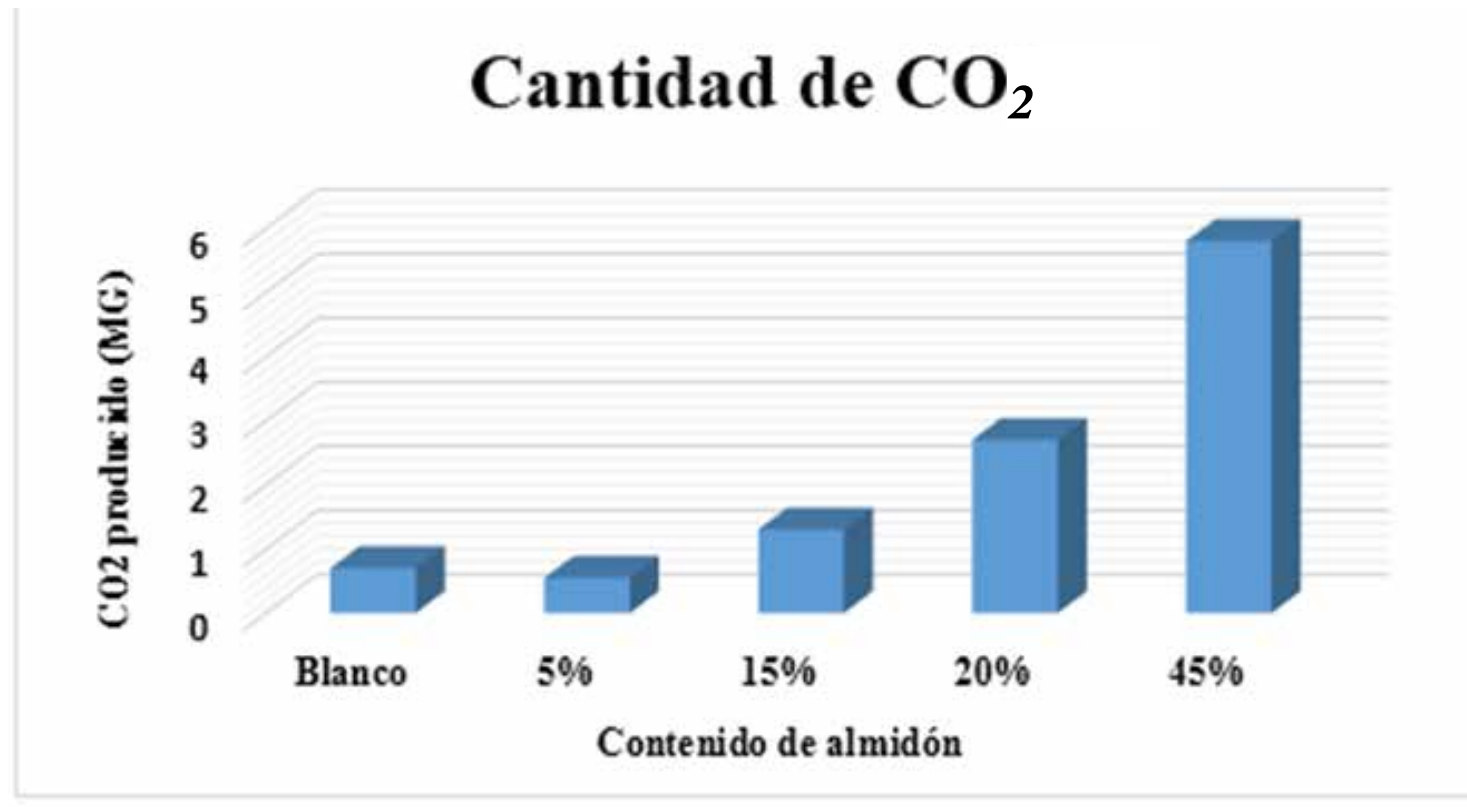

Figura 3: Producción de $\mathrm{CO}_{2}$ Fuente: Autor

\section{Conclusiones}

La investigación da como resultados un nuevo material compuesto biodegradable, que se puede transformar por los procesos de extrusión e inyección con parámetros ideales para la producción en serie de productos plásticos, siendo una alternativa para reemplazar los materiales sintéticos convencionales.

En la caracterización mecánica del material biodegradable por el ensayo de tensión, se observó que en el PEBD a mayor concentración de almidón sus propiedades mecánicas disminuyen.
Por su parte, en los ensayos de biodegradabilidad según la norma ASTM D-558 se comprobó que la producción de dióxido de carbono por los microorganismos presentes en el compostaje y analizado se incrementó, demostrando que el almidón de papa está siendo utilizado para llevar a cabo sus rutas metabólicas y respiratorias. Lo que comprueba la degradación del biodegradable.

De esta manera, y como resultado de los estudios realizados, es preciso afirmar que los materiales biodegradables con almidón de papa puede ser una alternativa para sustituir los polímeros sintéticos, contribuyendo a mejorar los problemas am- 
bientales existentes.

\section{Referencias}

Rodríguez L, Bello L, Yee H, González R (2013) Propiedades mecánicas y de barrera de películas elaboradas con harina de arroz y plátano reforzadas con nano partículas: Estudio con superficie de respuesta. Rev. Mexicana de Ingeniería Química. Vol. 12, No. 1.

Ortiz M., Villalobos M. (2013) Desarrollo de una película plástica a partir de almidón extraído de papa residual. Universidad Tecnológica del Suroeste de Guanajuato. México.

Rodríguez P., Camargo S., Villagrán I. (2016). Transformación y caracterización de un bioplástico por los por los procesos de extrusión e inyección. Revista Informador Técnico. Vol. 80, No. 2.

Villada H., Acosta H., Velasco R. (2006) Biopolímeros naturales usados en empaques biodegradables.

Muñoz E., Gómez E., Rodríguez P. (2015). Obtención y caracterización de un biodegradable a partir de almidón de papa y polietileno de baja por inyección. Revista Informador Técnico. ISSN: 2256-5035. Vol.79.

Rodríguez P., Camargo S. (2017). Caracterización de las propiedades mecánicas de un material biodegradable a partir de los procesos de extrusión y peletizado. Informador Técnico. ISSN: 2256-5035. Vol. 81, No.2

Rodríguez P., Camargo S., Cruz I. (2017), Desenvolvimento do processo de injeção com material biodegradável a partir da mistura de polietileno de baixa densidade e fécula de batata. Congresso ibero -americano de Engenharia Mecânica. Lisboa, Portugal ISSN: 978-989-95683.

Arévalo K. (1996). Elaboración de plásticos biodegradables a partir de polisacáridos y su estudio de biodegradación a nivel de laboratorio y campo. Universidad Autónoma de Nuevo León. México.

Vieyra Ruiz, Horacio. (2009). Elaboración de polímeros biodegradables polietileno- almidón y estudio de biodegradabilidad (Doctoral dissertation). Instituto Politécnico Nacional-México. 